\title{
Higienismo y medicina social: poderes de normalización y formas de sujeción de las clases populares
}

\section{Hygienism and social medicine: powers of normalization and means of subjecting the popular classes}

\author{
ANNA QUINTANAS
}

Universidad de Girona

Resumen. Partiendo de datos proporcionados por la historia de la medicina en España, quisiéramos mostrar que M. Foucault tenía razón cuando afirmaba que uno de los puntos neurálgicos, a partir de los cuales irradian los poderes de normalización en nuestra sociedad, es el de los discursos y las prácticas médicas. Concretamente, hemos centrado nuestro análisis en algunos de los textos más representativos del higienismo y la medicina social durante el siglo XIX y principios del siglo XX, para mostrar la enorme influencia que ejercieron dichos discursos y prácticas de la medicina sobre los cambios en el estilo de vida de las clases populares que se produjeron durante este período. Sin duda, la medicina - sus discursos, sus instituciones, sus prácticas, sus recetas, sus recomendaciones - ha desempeñado un papel protagonista en el diseño de los procesos de subjetivación a través de los cuales nos reconocemos y nos construimos como sujetos dotados de un determinado tipo de identidad.

Palabras clave: Poderes de normalización, procesos de subjetivación, biopolítica, higienismo, medicina social, historia de la medicina, Foucault.
ABstract. Based on data from the history of medicine in Spain, we would like to show that M. Foucault was right when he said that the discourses and practices of medicine are one of the key points from which the powers of normalization arise in our society. Specifically, we focus our analysis on some of the most representative texts of hygienism and social medicine during the nineteenth and early twentieth centuries, to show the enormous influence of these discourses and practices of medicine on changes in lifestyle of the popular classes that occurred during this period. Undoubtedly, the medicine - its discourses, its institutions, its practices, its prescriptions, its recommendations - has played a leading role in the design of the processes of subjectivation by means of which we recognise ourselves and construct ourselves as subjects possessing a specific type of identity.

Key words: Powers of normalization, processes of subjectivation, biopolitics, hygienism, social medicine, history of medicine, Foucault.

«La salud no es sólo la vida en el silencio de los órganos, es también la vida en la discreción de las relaciones sociales» ${ }^{1}$

G. Canguilhem 


\section{La «caja de herramientas» de M. Foucault}

Una de las aportaciones más interesantes del pensador francés M. Foucault (19261984) fueron sus investigaciones sobre las relaciones entre el saber y el poder en el ámbito de las ciencias humanas. Concretamente, la historia de la medicina fue un objeto de estudio privilegiado de su analítica del poder. Desde su primera obra, Folie et déraison. Histoire de la folie à l'âge classique (1961), hasta sus últimos textos sobre la Histoire de la sexualité (1976-1984), pasando por la Naissance de la clinique. Une archéologie du regard médical (1963), Foucault se interesó de forma destacada por el ámbito de la medicina. Para sustentar sus tesis, utilizó principalmente ejemplos de Francia, Inglaterra y Alemania. En este trabajo, pretendemos mostrar que el rico conglomerado de conceptos y herramientas aportado por Foucault, también puede ser utilizado en el caso de la historia de la medicina en España, siempre y cuando se tenga en cuenta que las periodizaciones pueden variar ligeramente debido al hecho de que, en este ámbito, como en tantos otros, España sufrió retrasos en comparación con otros países europeos.

Partiendo pues de datos proporcionados por los estudiosos de la historia de la medicina en España, ${ }^{2}$ quisiéramos mostrar que Foucault tenía razón cuando afirmaba que uno de los puntos neurálgicos de los que irradian los poderes de normalización en nuestra sociedad es el de los discursos y las prácticas médicas. Concretamente, hemos centrado nuestro análisis en algunos de los textos más representativos del higienismo y la medicina social durante el siglo XIX y principios del siglo $\mathrm{XX},{ }^{3}$ para mostrar la enorme influencia que ejercieron sobre los cambios en el estilo de vida de las clases populares que se produjeron durante este período.
Sin duda, la medicina - sus discursos, sus instituciones, sus prácticas, sus recetas, sus recomendaciones - ha desempeñado un papel protagonista en el diseño de los procesos de subjetivación a través de los cuales nos reconocemos y nos construimos como sujetos dotados de un determinado tipo de identidad.

\section{La cuestión social: salud, orden, riqueza y moral}

Durante el siglo XIX, a medida que avanzaban los efectos de la revolución industrial, se fue convirtiendo en una evidencia que el proletariado, como fuerza de trabajo, tenía sus límites, y que éstos debían ser respetados si no se quería menguar su potencia y su rentabilidad. ${ }^{4} \mathrm{De}$ esta forma, fue tomando protagonismo el problema de la salud del trabajador. ${ }^{5}$ Como señala Rodríguez Ocaña, el concepto de «enfermedad social», que implica relacionar el origen de la enfermedad con la propia organización social y especialmente con las desigualdades sociales, «no es un concepto inmanente, que estuviese presente desde los primeros momentos de la reflexión racional acerca de la naturaleza de las enfermedades, sino que se ha desarrollado en los dos últimos siglos, formando parte de la cultura industrial». ${ }^{6}$ Según este autor, la vinculación entre pobreza y enfermedad empezó a tener un carácter de evidencia a partir de los primeros decenios de siglo XIX, especialmente por la influencia de la aparición del cólera en Europa en 1831, así como también por la revolución de 1848 , que contribuyó al hecho de que los médicos mostraran más interés por las cuestiones sociales.

El higienismo y la medicina social, más allá de sus diferencias, tuvieron un elemento en común, que fue su interés por lo que entonces se llamó la «cuestión social», es decir, la preocupación por las condiciones de existencia de las clases 
más necesitadas. Si la medicina debía atender los problemas de salud, tenía que interesarse por la realidad cotidiana de las clases más desfavorecidas, puesto que de ella dependían en buena parte las enfermedades que sufría la población.

Desde esta preocupación por la «cuestión social», los médicos e higienistas intentaron convencer a los poderes públicos y a los patronos de que había buenas razones para invertir en la mejora de las condiciones de vida de los sectores más desfavorecidos de la sociedad. ${ }^{7}$ Méndez Álvaro, por ejemplo, en su obra De la actividad humana en sus relaciones con la salud y el Gobierno de los pueblos, indica al gobierno que, en los principios de la higiene, puede hallar soluciones, no sólo para mejorar el nivel de salud de la población, sino también para luchar contra los desórdenes sociales y las revueltas políticas, así como fórmulas para evitar el retraso económico del país. A los patronos, por su parte, se les intenta mostrar que la aplicación de las medidas de higiene pública provocaría un aumento en el rendimiento y la productividad de los trabajadores. ${ }^{8}$

En nombre del conocimiento privilegiado que el médico había adquirido sobre la población a través de su labor asistencial — que le permitía penetrar hasta el interior de sus viviendas y escudriñar en todas sus intimidades_-, se presentó a sí mismo como un experto en el arte de observar, corregir y mejorar el cuerpo social. El higienismo y la medicina social impulsaron al médico a ir más allá de su función de curar enfermedades, hasta convencerlo de que su saber le obligaba a jugar un papel protagonista en el seno de la sociedad y en el gobierno de la nación. Este vínculo esencial entre medicina y política queda bien resumido en el discurso que Mateo Seoane ${ }^{9}$ realizó en la Academia de Ciencias de Madrid en 1837, donde definió la higiene pública como el ámbito que debe incluir «todas las relaciones que las ciencias médicas tienen con el gobierno». La higiene pública consistiría en «el arreglo sistemático de los conocimientos médicos que concurren de un modo directo o indirecto a ilustrar la ciencia del gobierno». ${ }^{10}$

Entre las aportaciones que el saber médico podría proporcionar al gobernante, Seoane destacó la estadística médica. Tal como ya hacía tiempo que estaba sucediendo en países europeos más avanzados, las autoridades públicas podían utilizar sus datos, y su variada información sobre múltiples aspectos de las costumbres y el estilo de vida de la población, para buscar soluciones a problemas tan acuciantes como el de los altos índices de mortalidad y morbilidad. ${ }^{11}$

Así, según Seoane, la higiene pública no puede desvincularse de la política y de la economía, pero tampoco de la moral, puesto que consideraba que los consejos médicos también potenciaban la armonía entre las funciones físicas y los actos morales. El cuerpo no sería el único objetivo de las exhortaciones médicas, su influencia abarcaría también los hábitos y las costumbres de los individuos. De esta forma, los principios higiénicos servirían de norma para regular buena parte de la conducta de la población. Seoane puso el acento sobre todo en la necesidad de moldear el comportamiento de la mujer, puesto que de ella dependía, en buena medida, tanto la reproducción de la especie como la educación infantil. ${ }^{12}$

Desde este punto de vista, la salud, el orden, la riqueza y la moral conformaban un conglomerado unitario. En los tratados médicos del período era habitual encontrar la tesis de que la aplicación de los principios higiénicos, no sólo repercutiría en la mejora de las condiciones de salud de la población, sino también en la mejora de sus condiciones de vida y de trabajo, así como en la consolidación del orden político y en el buen funcionamiento 
de la economía. Como muy bien indica Rodríguez Ocaña, «dado que los tratadistas de primeros del siglo XIX habían cifrado la causa de estos males [criminalidad, mendicidad, prostitución, huelgas, revoluciones] en un sustrato individual alterado, de orden moral o "pasional", en la terminología de la época, no es extraño que las enfermedades sociales fuesen descritas por ciertos higienistas decimonónicos como verdaderas enfermedades morales que trascienden con su influencia al estado físico de los individuos al par que trastornan el organismo social». ${ }^{13}$ Aunque estos textos médicos subrayaban claramente la influencia de la organización social, y de las condiciones de vida y de trabajo, sobre la salud, no por ello se dejó de culpabilizar a los obreros de su situación, por los malos hábitos y las costumbres que dominaban su existencia cotidiana. Los tratados médicos hicieron, por tanto, un especial hincapié en la responsabilidad individual de los propios obreros.

Según Rodríguez Ocaña, aunque el primer tratado sistemático sobre las enfermedades del trabajo fue la obra de B. Ramazzini, Tratado de las enfermedades de los artesanos (Módena, 1700), es importante tener en cuenta la enorme influencia de la obra de Ch. Turner, Los efectos de las artes, comercios y profesiones, y del estado civil y hábitos de vida sobre la salud y la longevidad: con sugerencias sobre la eliminación de la mayor parte de los agentes que causan enfermedad y acortan la duración de la vida (1831), no sólo porque podría ser considerada la primera reflexión crítica sobre la Revolución Industrial realizada desde la medicina, sino también porque introdujo un elemento novedoso que no estaba presente en Ramazzini, y que acabó impregnando toda la literatura higienista del siglo XIX: la intención moralizante. ${ }^{14}$ Como indica González de Pablo, «desde finales del siglo XVIII y a lo largo del si- glo XIX, se produjo una inversión (...), la medicina y la higiene se transformaron en los determinantes de la moral y de la conducta.» ${ }^{15}$ A principios de la época moderna, la higiene había estado sometida a los principios de la religión, primero de forma dogmática y después intentando racionalizar su contenido, mientras que, en el paso del siglo XVIII al siglo XIX, tuvo lugar una inversión en los términos de esta ecuación. A partir de entonces, la propia medicina y la higiene se convirtieron ellas mismas en fuente de valores morales y de normas de conducta.

Como puede comprobarse en los textos de los higienistas españoles de esta época, el objetivo de esta medicina de carácter social debía ser precisamente conseguir un perfecto equilibrio entre salud, orden, riqueza y moral. Para conseguir este objetivo, los higienistas apelaban a la necesidad de reunir información y establecer consejos sobre todos y cada uno de los aspectos que influyen en la vida cotidiana de la clase trabajadora. Hacía falta supervisar sus viviendas, sus lugares de trabajo, los establecimientos que frecuentaban, pero también las actividades que realizaban en su tiempo de ocio, sus relaciones familiares, su alimentación, incluso el tipo de vestimenta que utilizaban. Se trataba de higienizar enseres, espacios y locales, pero también comportamientos, pasiones e instintos. Para el higienismo, luchar contra la prostitución, el juego, los vicios asociados a las tabernas, la promiscuidad dentro de las relaciones familiares, o fomentar el orden, la disciplina, la limpieza, los hábitos saludables, y el ahorro, servía tanto para mejorar la salud y el bienestar de la población, como para aumentar la productividad económica y asentar el orden público. ${ }^{16}$

\section{El valor económico de la vida humana}

Uno de los argumentos más utilizados por los higienistas y por la medicina so- 
cial para convencer a los poderes públicos de la necesidad de invertir en el bienestar de la población, consistió en destacar el valor económico de la salud. Son habituales, en los tratados de la época, los cálculos sobre los costos y las pérdidas debidos a las enfermedades y las muertes evitables. En este sentido, es ilustrativa la obra Madrid bajo el punto de vista médico-social (1902), de Ph. Hauser (18321925), uno de los higienistas más relevantes que vivió en España los últimos cincuenta años de su vida. Hauser intenta argumentar sobre la necesidad del Estado de invertir en sanidad. ${ }^{17}$ Pone ejemplos de ciudades europeas como París, Londres o Berlín, donde ya habrían fructificado los esfuerzos realizados para mejorar las condiciones sanitarias de esas ciudades, dando como resultado un descenso notable de la mortalidad y la morbilidad, que tanto gasto producen a las arcas públicas. Hauser indica cómo se calcula el valor económico de la vida humana de la forma siguiente:

«Este valor económico representa lo que cada individuo ha costado a su familia para vivir, desarrollarse e instruirse, pues es un empréstito que se hace al capital social hasta que se llegue a la edad en que el hombre adquiere fuerzas morales y físicas que le posibilitan para ganar su sustento por medio del trabajo y para rembolsar lo que ha gastado. Los higienistas ingleses y americanos que se han ocupado de esta cuestión estiman distintamente el valor de un hombre adulto. Chadwick estimaba en 5.000 francos el valor general de un trabajador llegado a la edad del adulto. Estimando en dos francos por día el precio de su trabajo y en trescientos días el trabajo de un año, resultan 600 francos anuales, los cuales, capitalizados según la tasa de las rentas vitalicias, dan 6.000 francos. Como hay trabajadores que ganan hasta 10 francos al día, no se puede admitir un tipo igual para todos los trabajadores; pero tomando un término medio, serían 10.000 francos por individuo, y siendo 5.000 individuos el exceso de mortalidad, éstos representan 50 millones de pérdida anual; aun- que de esta cifra hay que deducir el número de viejos y mujeres que no pertenecen a la clase productiva; pero aun así puede considerarse que ese exceso de mortalidad evitable, representa una pérdida de 15 a 20 millones anuales para el Estado y la familia, sólo en Madrid.

Además del exceso de mortalidad, hay todavía que tener en cuenta el número desproporcionado de enfermos por dolencias infecciosas que obligan al obrero a abandonar el trabajo; pues 5.000 de exceso de mortalidad anual representa un mínimum de 35 a 40.000 enfermos, que, contando sólo diez días de enfermedad por término medio, para cada uno, forma en conjunto 350.000 días sin trabajo, que son 700.000 francos de pérdida, y a esto hay que agregar los gastos que traen la enfermedad o la muerte.» ${ }^{18}$

El mismo tipo de cálculo de la vida humana podemos encontrarlo, por ejemplo, en A. Espina, en una obra donde aboga a favor de una ley de seguro obligatorio en caso de invalidez:

«Calculando, con la mayoría de los sociólogos, el valor de la vida de un obrero manual en 5.000 pesetas, como término medio, y habiendo muerto, por un promedio, en cinco años, 448.996, se puede ver el gran valor de una legislación pronta y rápidamente discutida, que evite esta sangría suelta a España en personas y en producción, legislación que, si se hiciera con el empeño y la premura que un encasillado de representantes, y pusiéramos igual empeño en hacerla que en conseguir un distrito, muy pronto nuestra nación se pondría de un salto, pues a paso de hombre no es posible, al nivel de las cultas e higiénicas. Ved, señores, en cifra redonda, calculando al entero, que perdemos 5.000.000.000 de pesetas, y bien podemos todos hacer un esfuerzo para menguar siquiera tres de los últimos ceros del horrible decimal que os presento. Pero si del hombre muerto se deduce tal cantidad, hay que ver cuán grande no será el decimal que resultará de las enfermedades que podemos evitar y cuán grande es el ahorro con sólo ahorrar un día de enfermedad con el socorro y la asistencia a tiempo bien dirigida.» ${ }^{19}$

O también puede verse en las siguientes observaciones de F. Murillo Palacios: 
«Obedeciendo las desventuras del proletariado a la falta de recursos, la panacea no puede ser otra que proporcionarle los suficientes para levantar su dietario al nivel de la línea de pobreza, lo cual supone el equilibrio entre los ingresos y los gastos de primera necesidad con el aditamento de un margen o arreglo que le permita conllevar los infortunios y azares de la vida.

Anteriormente dejo demostrado que la penuria de medios con sus inevitables secuelas de alimentación insuficiente, vivienda insalubre, fatiga prematura y desplazamiento de la mujer y de los hijos, engendran la enfermedad en la familia obrera, la degeneración de la raza y la disminución de la capacidad productora colectiva, lo que, en último término, representa una pérdida cuantiosa del capital nacional en vidas y haciendas. Hay, pues, tres elementos interesados en la resolución del problema: el obrero que pierde la salud y la existencia, el patrono que no obtiene del capital el debido rendimiento y el Estado que, nutriéndose de una y otra raíz, no puede prosperar y engrandecerse si ambas caducan. Los tres necesitan colaborar en la obra común, porque los tres son, en definitiva, los beneficiarios directos del provecho.» 20

Los ejemplos en este sentido serían muchos. B. Avilés, en Estudio sobre el valor económico de la vida y la salud (1889), calculaba en unos 35.670 millones de pesetas el valor de la población total de España, con una media por habitante de 2.100 pesetas, cifra que consideraba justificada, entre otros argumentos, por estar en consonancia con el precio de la exención del servicio militar, oscilando entre 1.500 y 2.500 pesetas. Los gastos correspondientes a la enfermedad, junto con el coste del entierro y la sepultura, los calculaba en más de 545 millones, 160 de los cuales corresponderían a enfermedades infecciosas. A. Larra y Cerezo, por su parte, en Los grandes problemas higiénicos y sociales en relación con las instituciones armadas (1902), calculaba que el recluta tenía un valor de 2.000 pesetas, que consideraba que podría in- crementarse hasta 5.000 si se le enseñaba a leer y a escribir durante la instrucción.

\section{La gestión médica de las patologías sociales}

Estos cálculos económicos sobre la vida humana tenían como objetivo convencer a los poderes públicos y a los patronos de que la mejora de las condiciones de vida y de trabajo de la población, comportaría tanto el aumento del nivel de salud del individuo como la erradicación de las llamadas «patologías sociales». La sociedad era concebida como un organismo vivo, con sus propias características anatómicas y fisiológicas. Las patologías sociales serían todas aquellas alteraciones del orden político, económico y moral vigente que se consideraba que, de alguna manera, perjudicaban el estado de salud de la población: desde el alcoholismo, el nicotismo, y la prostitución, hasta la mendicidad, la vagancia, la criminalidad, el suicidio, la ignorancia o el fanatismo, pasando por el caciquismo, el pauperismo, las huelgas, los motines y las revoluciones. En todas estas situaciones, se entendía que se estaba rompiendo el equilibrio necesario para conservar la salud, tanto del organismo social como del individual. Como afirmaba F. Rubio Galí, los individuos serían como las células de la sociedad, es decir, de un cuerpo colectivo altamente complejo, que necesita, para su estudio, de la socio-patología - una ciencia esencialmente médica, pero que se complementaba con otras ramas del saber como la filosofía, la moral, la religión y el derecho. Como ejemplos de patología social, Rubio Galí pone los siguientes:

"Vicios individuales trascendentes a la familia y a la sociedad.- Alcoholismo, nicotismo, nepentismo, mendicidad, egoísmo, vagancia, prostitución, criminalidad.

Vicios sociales. - Preocupaciones, ignorancia, fanatismo, sensualismo, caudillaje, caciquismo, parasitismo, pauperismo. 
Contagios psíquicos. - Neuropatías colectivas y sociales; antropofagomanía.

Trastornos funcionales. - Huelgas, motines, revoluciones.» ${ }^{21}$

En esta línea, donde confluye el gobierno de la población y la gestión de las enfermedades, se abogó por el hecho de que fuera la figura del mismo médico la que dirigiera todo el proceso de regeneración, de cambio y de mejora de la sociedad. A. Aguado y L. Huerta, por ejemplo, declaraban con convencimiento lo siguiente:

«Pero la dirección de este régimen no puede encomendarse ya a los fracasados políticos, diplomáticos y leguleyos, desconocedores de la fisiología y de la psicología del hombre y de las necesidades reales de la humanidad. Este régimen cae exclusivamente bajo los auspicios de una nueva ciencia, de la MEDICINA SOCIAL, que es la Medicina, la Pedagogía, la Sociología y la Jurisprudencia íntimamente unidas. Sólo los médicos sociólogos ejercerán en lo sucesivo el directo y valioso influjo social capaz de mejorar al ser humano y hacerle fácil su marcha terrena hacia el destino inmortal.» 22

Para una tarea tan trascendental, Aguado y Huerta apostaron por la creación de un «poder sanitario», que definieron de la forma siguiente:

«Si la Medicina social ha de ser una realidad, precisa la transformación completa de nuestra política sanitaria, una remoción de los actuales organismos administrativos, la creación de órganos adecuados para la suprema función de garantizar la salud pública, primer deber de los Estados modernos y el más sagrado derecho individual que ha de consagrarse en las futuras constituciones políticas, $y$, en suma, la afirmación de la existencia de un nuevo poder del Estado, independiente, desprendido de la función ejecutiva, pero autónomo, rápido y decisivo, de tal suerte, que sea la vanguardia de la sociedad organizada contra las invasiones del destructor ejército que atenta contra la salud pública: el poder sanitario.» 23
Según su opinión, este «poder sanitario» debía tener un ámbito de actuación prácticamente infinito:

«Cae de lleno bajo su dominio científico el problema de las subsistencias, el de los salarios, el de las viviendas higiénicas y las ciudades-jardines, el de saneamiento y urbanización de los pueblos, el de los parques nacionales, el de la asistencia y educación de los niños, el de la protección a las madres, el del trabajo en el campo, fábricas y oficinas, las plagas sociales, la reforma penitenciaria, las luchas sociales... Todo es atendido por ella cuando se trata de garantizar la salud y mejorar la vida de la humanidad.

Desde el aire que respira y la tierra que pisa, hasta las costumbres, las pasiones que le dominan, las mismas ideas, aspiraciones, empresas que acomete, todo, en fin, cuanto integra el medio ambiente en que desenvuelve su existencia, influye de modo poderoso en la salud, en la vida del hombre, como individuo y como especie, y es objeto de estudio delicado por parte de la Medicina social que tiende a disponerlo todo para el logro del ideal supremo, del único verdaderamente positivo y compendio de todos los demás: VIVIR MUCHO Y VIVIR BIEN.» ${ }^{24}$

Por su parte, M. Iglesias Carral, que fue inspector provincial de Sanidad, en El médico social (1916), habla de la tarea del médico social como de «una obra de catequesis», que tendría como principal objetivo predicar a la población sobre «el concepto firme de su deber sanitario». El médico social debería utilizar todos los medios propagandísticos hasta conseguir que la población llegara a asimilar «la pauta higiénica a que previsoramente ha de sujetarse la vida». Y de la misma forma que el sacerdote debía cuidar sobre todo del pecador, la medicina social debía velar especialmente por la clase trabajadora, puesto que llevaba un estilo de vida más desordenado e indisciplinado. Sin olvidar que el médico social, convertido ahora en «el tutor científico del obrero», según Iglesias Carral, debería partir 
siempre de la premisa que relaciona necesariamente salud y productividad:

«En la enfermedad como en el accidente, será la aspiración del médico social restituir al que sufre la máxima capacidad de trabajo en el menor tiempo, poniendo para ello a contribución toda clase de medios que logren el resultado apetecido, y cuando no se consiga lo deseado, adaptar el grado de aptitud que reste a la modalidad de trabajo más conveniente.» 25

En este sentido, resulta significativa la función que Monlau atribuye a las escuelas dominicales, que debían ser creadas para evitar que la clase trabajadora empleara su tiempo libre de forma perniciosa. Las escuelas dominicales, incluidas dentro de las medidas higiénico-moralizadoras, tenían que colaborar en la instrucción de la clase trabajadora, hasta conseguir que ésta interiorizara los siguientes principios:

«No hay felicidad posible fuera del camino de la virtud; que la desigualdad en los bienes de fortuna está tan en la naturaleza como la desigualdad de talento, de talla, de robustez o de fuerzas físicas; que sin jerarquías no hay orden ni sociedad posibles; que la templanza y la moderación en todo es la condición de la buena salud; que es un absurdo ridículo querer fijar un mínimum de jornal y un máximum de trabajo; que la fórmula esencial del progreso económico es producir cada día más, cada día más pronto, y cada día más barato; (...) que las coaliciones de obreros para hacer subir el precio del jornal son recursos ya gastados, estériles y ridículos; que el aumento forzado del precio del jornal es insostenible; (...) que las agresiones brutales, los crímenes que alguna vez han manchado de sangre el recinto mismo de las fábricas o de los talleres, son puras manifestaciones de una ira salvaje e impotente, que al cabo es siempre reprimida y castigada con fuerte daño del criminal agresor; que hay, en fin, principios eternos de moral a cuyas consecuencias están indeclinablemente sujetos así el fabricante como el obrero. (...)

Todo eso, y todo cuanto a su bienestar e instrucción conduzca, comprenderá perfecta- mente el obrero, si se le explica con claridad y amor. No hay, pues, para qué insistir más en la utilidad de las escuelas que tan bellos resultados pueden proporcionar.» ${ }^{26}$

Aunque, $\mathrm{Ph}$. Hauser, consciente del hecho de que la clase trabajadora no aceptaría sin más el papel de tutor que el médico pretendía ejercer sobre su estilo de vida, recomendó que los principios de la higiene social se fueran introduciendo paulatinamente desde diversos flancos, hasta que llegaran realmente a convertirse en familiares para todo el mundo. Para ello aconsejó la presencia de médicos inspectores, con tareas divulgativas, en escuelas, cuarteles, hospitales, asilos y hospicios; así como en establecimientos colectivos como teatros, cafés, iglesias y talleres:

«Es necesario que el hombre, en todas las fases de su existencia, vea, oiga y toque prácticamente los mandamientos sagrados de la higiene; es decir, que esta ciencia de la salud y de la vida penetre en su mente por todos los sentidos. Una vez que las masas populares hayan adquirido los hábitos de la limpieza y hayan comprendido la influencia salutífera de la higiene, el médico no encontrará ya dificultad en su clientela para hacer comprender a las familias su deber profesional para con la sociedad.» 27

Todos los esfuerzos eran pocos teniendo en cuenta que, en último término, se consideraba que la interiorización de los mandamientos higiénicos lograría la transformación integral del ser humano:

«El nuevo tipo de hombre normal tendría el cuerpo de Adonis, la inteligencia de Aristóteles y el corazón de Francisco de Asís. Y el nuevo modelo de mujer perfecta tendría el cuerpo de Venus, el talento de Hipatía y el corazón de María. Con estos ejemplos sólo intentamos hacer bien sensible el supremo anhelo de la obra augusta del perfeccionamiento humano. Y esto es lo que pretende, en suma, la Medicina social.» 28 
Higienismo y medicina social ...

\section{Lo normal y lo patológico: cuestiones de biopolítica}

Partiendo del título del célebre libro de G. Canguilhem ${ }^{29}$ - que tanta influencia ejerció sobre Foucault por el hecho de cuestionar la pretendida oposición entre lo «normal»y lo «patológico»-, podemos concluir que el higienismo y la medicina social, tal como se desarrollaron en España en el siglo XIX y principios del siglo XX, pueden ejemplificar la tesis foucaultiana de los poderes de normalización inherentes a la biopolítica. ${ }^{30}$ La biopolítica, según Foucault, caracterizaría la nueva forma de gestión de la vida humana propia de la sociedad contemporánea. Si durante el Antiguo Régimen el arte de gobernar se caracterizó sobre todo por el hecho de que el poder absoluto disponía del derecho de matar, la racionalidad política contemporánea habría desplazado el acento hacia el derecho o la capacidad de administrar la vida. Nuestra modernidad, según Foucault, se distinguiría precisamente por haber posibilitado la entrada de la «vida» en la historia política. El bio-poder se definiría no tanto por su capacidad de matar, como por el hecho de disponer de los mecanismos necesarios para poder invadir la vida globalmente. En el momento en que se pasó de una sociedad del castigo y del suplicio, a una sociedad con predominio de técnicas disciplinarias y reguladoras, los mecanismos del poder se empezaron a distinguir no tanto por su capacidad de doblegar, obstaculizar o destruir las fuerzas que someten a su ejercicio, como por sus funciones de producción, de incitación, de reforzamiento o de reorganización de dichas fuerzas.

Según Foucault, en nuestra sociedad, la medicina formaría parte del dispositivo que dibujan las actuales formas de gobierno de la población. En una sociedad normalizadora como la nuestra, lo médi- co juega un papel fundamental en el diseño de la partición entre lo «normal» y lo «patológico». Las actuales formas de conducción de las conductas de las personas atraviesan nuestro cuerpo:

«El control de la sociedad sobre los individuos no se efectúa sólo por la conciencia o la ideología, sino también en el cuerpo y con el cuerpo. Para la sociedad capitalista lo que importa ante todo es la biopolítica, la biología, lo somático, lo corporal. El cuerpo es una realidad biopolítica; la medicina es una estrategia biopolítica.» ${ }^{31}$

Los mecanismos del biopoder, según Foucault, se habrían organizado formando dos vectores principales. Por un lado, las disciplinas del cuerpo - cuyas huellas pueden rastrearse ya a partir del siglo XVII-, y que han dado lugar a toda una «anatomopolítica del cuerpo humano», que ha tratado al cuerpo como una máquina cuyas fuerzas y habilidades deben ser canalizadas hasta conseguir su grado máximo de utilidad y docilidad. Por otro lado, toda una «biopolítica de la población» — surgida un poco más tarde, a mediados del siglo XVIII-, y que ha pretendido regular al cuerpo-especie, a la población, a los procesos biológicos contemplados como conjunto (la natalidad, la mortalidad, la morbilidad, el nivel de salud, la demografía). El papel central que jugaría la medicina dentro de la biopolítica sería precisamente gracias a su función de bisagra entre estos dos vectores, puesto que su ámbito de actuación incluye tanto al cuerpo del individuo (cuerpo-organismo) como al «cuerpo» de la población (cuerpo-especie).

La biopolítica, como forma de gestión calculadora de la vida, ha dado lugar a todo un nuevo conglomerado de saber-poder, en cuya construcción la medicina ha hecho contribuciones esenciales. La racionalidad política característica de la biopolítica, ha ido acompañada por la creación paralela de todo un saber sobre 
los individuos y sobre la población (estadísticas sobre mortalidad, morbilidad, natalidad, longevidad, sexualidad, migraciones, accidentes), ${ }^{32}$ y por la puesta en funcionamiento de todo un conjunto de mecanismos que permiten supervisar, gestionar y regular la existencia de los individuos, mientras se está pretendiendo mejorar sus condiciones materiales de vida (medidas de salud preventiva, disposiciones sobre seguridad laboral o sobre seguridad vial, normas para la gestión de riesgos, campañas de salud pública). Tal como hemos podido observar en el contenido de los textos del higienismo y de la medicina social, las relaciones de poder en nuestras sociedades se definen por este tipo de «positividad», por actuar más desde el incentivo que desde la represión, más desde los consejos y la publicidad, que desde la fuerza de la violencia física. De esta forma resume González de Pablo este proceso a través del cual la promoción de la salud, como fuente de determinadas normas morales y de conducta, se ha ido convirtiendo no sólo en un mandato impuesto desde arriba por distintas instancias de poder, sino, finalmente, tam- bién, en una demanda exigida por la propia población, una vez ésta ha asimilado e interiorizado sus exigencias:

«Pero las progresivas demandas de la sociedad industrial desarrollada forzaron un nuevo acercamiento a la consideración de la salud con el fin de llegar a grandes capas de población a aceptar el moderno sistema de vida. Por ello, la colonización desde arriba debió acompañarse de un progresivo proceso de asimilación desde abajo en la aceptación de la nueva forma de consideración de la salud. Para que esta asimilación se produjera con la suficiente extensión e intensidad se precisaba, primero, una noción de salud y un proceder para su conservación que fueran tenidos por la población como absolutamente científicos y, por tanto, cualquier tipo de aditamento moral presente en ellos pasara del plano real al ideal; y, segundo, que su aplicación a la esfera pública se hiciera no mediante procederes caritativos sino a través de una política científicamente concebida. Mediante la adquisición de estos ropajes científicos aparentemente neutros (el científico-natural primero y el científico-social después), la higiene se impondría por la fuerza de la objetividad de forma irresistible al ser no sólo exigida desde arriba sino también demandada desde abajo, con el consiguiente efecto de autocontrol de las conductas.» ${ }^{33}$

\section{NOTAS}

1 Canguilhem, G., La santé. Concept vulgaire et question philosophique, París, Ed. Sables, 1990, pp. 27-28.

2 En este sentido nos han sido especialmente útiles los siguientes textos: Rodríguez Ocaña, E., «El concepto social de enfermedad», en Albarracín, A. (coord.), Historia de la enfermedad, Madrid, Centro de Estudios Wellcome-España, 1987, pp. 340-349; Rodríguez Ocaña, E., La constitución de la medicina social como disciplina en España (1882-1923), Madrid, Ministerio de Sanidad y Consumo, 1987; González de Pablo, A., «Sobre la configuración del modelo de pensamiento de la higiene actual: el caso español», DYNAMIS. Acta Hisp. Med. Sci. Hist. Illus., 15, 1995, pp. 267-299.

3 Se han utilizado sobre todo las recopilaciones de textos médicos de este período incluidas en López Piñero, J. M., M. Seoane. La introducción en España del sistema sanitario liberal (1791-1870), Madrid, Ministerio de Sanidad y Consumo, 1984; y Rodríguez Ocaña, E., La constitución de la medicina social, cit.

${ }^{4} C f$. Montiel, L., «Beneficios y riesgos de un nuevo valor: el valor salud en la sociedad postindustrial», en Montiel, L. (coord.), La salud en el estado de bienestar. Análisis histórico, Madrid, Ed. Complutense, 1993, pp. 37-52. Siguiendo las tesis de G. Canguilhem, Montiel destaca que el término «salud» es de hecho un «concepto vulgar» y que difícilmente la ciencia puede ofrecer una definición positiva y objetiva de su significado. No obstante, a lo largo de los dos últimos siglos, la salud se ha llegado a definir positivamente, pero sólo al precio de relacionarla inevitablemente con la productividad. $C f$. Canguilhem, G., La santé, cit.; y también la definición de salud de la célebre conferencia de la OMS de 1978, en AlmaAta: «Uno de los principales objetivos de los gobier- 
Higienismo y medicina social ...

nos, de las organizaciones internacionales y de la comunidad mundial entera en el curso de los próximos decenios debe ser el que todos los pueblos del mundo alcancen en el año 2000 un nivel de salud que les permita llevar una vida social y económicamente productiva» («Declaración de Alma-Ata», en Actividades de la OMS 1978-1979. Informe Bienal del Director General, Ginebra, 1980, p. 8).

5 Sobre la preocupación de la medicina española por la salud del trabajador durante el siglo XIX, $c f$. López Piñero, J. M., «El testimonio de los médicos españoles del siglo XIX acerca de la sociedad de su tiempo. El proletariado industrial», en López Piñero, J. M.; García Ballester, L. y Faus, P., Medicina y sociedad en la España del siglo XIX, Madrid, Sociedad de Estudios y Publicaciones, 1964, pp. 109-208. Y también Rodríguez Ocaña, E., «Paz, trabajo, higiene. Los enunciados acerca de la Higiene industrial en la España del siglo XIX», en Huertas, R. y Campos, R. (eds.), Medicina Social y Movimiento Obrero en España (siglos XIX y XX), Madrid, F.I.M., 1992, pp. 383-406.

${ }^{6}$ Rodríguez Ocaña, E., «El concepto social de enfermedad», cit., p. 341.

$7 C f$., por ejemplo, los comentarios de J. Font i Mosella, en Consideraciones sobre los inconvenientes que irrogan a la salud de los jornaleros y a la pública de Barcelona las fábricas y en especial las de vapor, y sobre las ventajas de trasladarlas a la llanura de Casa Túnez (Barcelona, 1852), sobre el hecho de que las malas condiciones de vida y de trabajo del obrero barcelonés repercuten negativamente en el aprovechamiento de su fuerza laboral.

8 Cf. Méndez Álvaro, F., De la actividad humana en sus relaciones con la salud y el Gobierno de los pueblos, Madrid, 1864, p. 17. Según Méndez Álvaro, las pésimas condiciones de existencia de los obreros provocan no sólo la disminución de la población, sino también la mala calidad de la que logra sobrevivir, lo cual conduce inevitablemente a la decadencia de los Estados, puesto que una población debilitada, cuando no imposibilitada, para el trabajo, poco puede contribuir al progreso económico.

9 El doctor Mateo Seoane (1791-1870), seguidor del utilitarismo benthamista, y defensor del movimiento liberal avanzado, fue uno de los higienistas más reputados. Seoane fue el maestro de P. F. Monlau y F. Méndez Álvaro, dos de los médicos de más influencia en España durante los años centrales del siglo XIX. Pedro Felipe Monlau (1808-1871) tuvo una gran producción científica y divulgadora. Sus obras sobre higiene pública y privada tuvieron numerosas ediciones durante el siglo XIX. Francisco Méndez Álvaro (1806-1883) tuvo una producción literaria más limitada, pero también dejó su huella en los ambientes médicos y en el de gobierno, donde ocupó cargos importantes. $C f$. López Piñero, J. M., M. Seoane, cit.

10 Cf. Seoane, M., «Principios en que deben fundarse las medidas legislativas y administrativas en todo lo concerniente a higiene pública», en López Piñero, J. M., M. Seoane, cit., p. 176.

11 En España, en comparación con otros países europeos como Francia o Inglaterra, el desarrollo de la estadística médica fue mucho más lento. En Barcelona, desde 1877, y en España, a partir de 1879, hubo un primer intento serio de producción sistemática de estadísticas de población con fines sanitarios gracias a las disposiciones impulsadas por Cástor Ibáñez de Aldecoa como Gobernador civil y, después, como Director general de Beneficencia y Sanidad. Pero, sólo la publicación anual, a partir de 1902, de Movimiento de la población de España permitió la realización de análisis reglados con una mínima seriedad estadística.

12 Cf. Seoane, M., «Consideraciones generales sobre la estadística médica», 1838 . Texto reproducido en J. M. López Piñero, M. Seoane, cit., pp. 187-212. Sobre la paulatina introducción del control médico en relación a la maternidad y a la infancia, $c f$. Rodríguez Ocaña, E. y Perdiguero, E., «Science and social persuasion in the medicalization of childhood in 19thand 20th - Century Spain», História-Ciências, Saúde-Manguinhos, v. 13, n. ${ }^{\circ}$ 2, pp. 303-324, abril-junio 2006.

13 Rodríguez Ocaña, E., «El concepto social de enfermedad», cit., p. 345 .

$14 C f$. Rodríguez Ocaña, E., «El concepto social de enfermedad», cit., p. 342.

15 Cf. González de Pablo, A., «Sobre la configuración del modelo de pensamiento de la higiene actual: el caso español», cit., pp. 267-299. En este sugerente estudio, se muestra cómo la promoción de la salud, especialmente a partir de la industrialización, ha estado íntimamente relacionada con el fomento de determinadas formas de conducta, adecuadas a las necesidades socio-económicas del período histórico. La citación pertenece a la p. 279.

16 Sobre la frecuente reivindicación de los higienistas españoles del siglo XIX de su papel de auxiliares privilegiados del gobierno, y sus propuestas para encuadrar y normativizar el comportamiento de la clase trabajadora, resulta especialmente interesante el siguiente texto: Campos Marín, R., «La sociedad enferma: Higiene y Moral en España en la segunda mitad del siglo XIX y principios del siglo XX», Hispania. Revista española de historia, LV/3, n. ${ }^{\circ} 191,1995$, pp. 1093-1112.

17 En relación a los trabajos de Hauser, Ocaña y Martínez constatan que «sus estudios sobre la salud de las ciudades parten siempre de la cuantificación de los fallecimientos, a la que asigna una dimensión económica. Su interpretación de la sobremortalidad en relación con la contaminación del subsuelo y los defectos de la vida urbana exigía que los poderes públicos, interesados en la preservación e incremento de la riqueza nacional, interviniesen para conseguir un saneamiento completo y el desarrollo de planes higiénicos de reforma urbana. Ésta era la vía teórica por la que la higiene se convertía en política.» $C f$. Rodríguez Oca- 
ña, E. y Martínez, F., Salud Pública en España. De la Edad Media al siglo XXI, Granada, Escuela Andaluza de Salud Pública, 2008, pp. 36-37.

18 Hauser, Ph., Madrid bajo el punto de vista médico-social (Madrid, 1902). La introducción de esta obra se haya reproducida en Rodríguez Ocaña, E., $L a$ constitución de la medicina social, cit., pp. 83-96. La citación pertenece a las pp. 89-90.

En el texto, Hauser se refiere a E. Chadwick (1800-1890), abogado inglés que perteneció al círculo de J. Bentham, y que fue uno de los impulsores del movimiento sanitario británico y un estimulador de la intervención estatal en el ámbito sanitario a través de sus críticas a la legislación sobre beneficencia. Especialmente importante fue su obra de 1842, Report on the sanitary condition of the labouring population of Great Britain.

19 Espina Capó, A., «El seguro de la invalidez» (Madrid, 1917), en Rodríguez Ocaña, E., La constitución de la medicina social, cit., p. 109.

20 Murillo Palacios, F., «La defensa social de la salud pública» (Madrid, 1918), en Rodríguez Ocaña, E., La constitución de la medicina social, cit., p. 149.

21 Rubio Galí, F., «La Socio-Patología» (Madrid, 1890), en Rodríguez Ocaña, E., La constitución de la medicina social, cit., p. 212.

22 Aguado Marinoni, A. y Huerta, L., «Cartilla popular de Medicina Social» (Madrid, 1919), en Rodríguez Ocaña, E., La constitución de la medicina social, cit., p. 244.

23 Ibid., pp. 259-260.

24 Ibid., p. 249.

25 Iglesias Carral, M., «El médico social» (1916). Texto reproducido en Rodríguez Ocaña, E., La constitución de la medicina social, cit., pp. 231-240. La citación pertenece a la p. 233.

26 Monlau, P. F., «Higiene industrial» (1856), en Monlau, P. F. y Salarich, J., Condiciones de vida y tra- bajo obrero en España a mediados del siglo XIX, Barcelona, Anthropos, 1984, pp. 103-104. Según algunos estudiosos, Monlau sería uno de los autores españoles donde se muestra más claramente cómo la higiene de este período se caracterizó por su voluntad de ofrecer coordenadas tanto para el ordenamiento moral individual como para el social. Cf. Granjel, M., Pedro Felipe Monlau y la higiene española del siglo XIX, Salamanca, Cátedra de Historia de la Medicina de la Universidad de Salamanca, 1983, p. 103; y González de Pablo, A., «Sobre la configuración del modelo de pensamiento de la higiene actual: el caso español», cit., pp. 283-285.

27 Hauser, Ph., Madrid bajo el punto de vista médico-social, cit., pp. 91-92.

28 Aguado Marinoni, A., y Huerta, L., «Cartilla popular de Medicina Social», cit., p. 244.

29 Cf. Canguilhem, G., Le normale et le pathologique, París, P.U.F., 1966.

$30 \mathrm{Cf}$. Foucault, M., «Derecho de muerte y poder sobre la vida», en Historia de la sexualidad I. La voluntad de saber, Madrid, Siglo XXI, 1989, pp. 161194; y la trascripción de la undécima lección del curso del Collège de France impartido por Foucault entre finales de 1975 y principios de 1976, «Del poder de soberanía al poder sobre la vida», en Genealogía del racismo. De la guerra de las razas al racismo de Estado, Madrid, Ediciones La Piqueta, 1992, pp. 247-273.

31 Foucault, M., «La naissance de la médecine sociale», en Dits et écrits, vol. II, París, Gallimard, 2001, p. 210.

32 Cf. Foucault, M., «Naissance de la biopolitique», en Dits et écrits, vol. II, París, Gallimard, 2001, p. 818 .

33 González de Pablo, A., «Sobre la configuración del modelo de pensamiento de la higiene actual: el caso español», cit., p. 286. 